\title{
Carbon C 14 Telotristat Etiprate
}

National Cancer Institute

\section{Source}

National Cancer Institute. Carbon C 14 Telotristat Etiprate. NCI Thesaurus. Code C111891.

An orally bioavailable, tryptophan hydroxylase (TPH) inhibitor prodrug labeled with carbon C 14, which could be used to evaluate the pharmacokinetic profile of telotristat etiprate. Upon administration, telotristat etiprate is converted to its active moiety, telotristat (LP778902), which binds to and blocks the activity of TPH. This may result in a reduction in peripheral serotonin $(5-\mathrm{HT})$ production and improvement of serotonin-mediated gastrointestinal adverse side effects, such as severe diarrhea. TPH, the rate-limiting enzyme in serotonin biosynthesis, is overexpressed in carcinoid tumor cells. Telotristat radiolabeled with carbon C 14 facilitates the evaluation of the pharmacokinetic characteristics of this agent, including its absorption, distribution, metabolism, and excretion (ADME). 